\title{
REMOVAL OF HIGH DENSITY IMPULSE NOISE USING MORPHOLOGICAL BASED ADAPTIVE UNSYMMETRICAL TRIMMED MID-POINT FILTER
}

\author{
${ }^{1}$ Saravanakumar, S., ${ }^{2}$ A. Ebenezer Jeyakumar and ${ }^{3}$ K.N. Vijeyakumar \\ ${ }^{1,3}$ Department of Electronics and Communication Engineering, \\ Anna University Regional Centre, Coimbatore, India \\ ${ }^{2}$ Director Academics, Sri Ramakrishna Engineering College, Coimbatore, India
}

Received 2013-10-08; Revised 2013-10-21; Accepted 2014-03-01

\begin{abstract}
A Morphological based Adaptive Unsymmetrical Trimmed Mid-Point Filter (MAUTMPF) for the restoration of gray scale images corrupted by salt and pepper noise for varying noise densities is proposed in this study. Images corrupted by impulsive noise severely hinder subsequent image processing tasks, such as edge detection, image segmentation, object recognition, etc. Therefore, it is absolutely essential to restore the original image from the corrupted image. The proposed algorithm replaces the corrupted pixel by mid point value out of the retained pixels other than 0 's and 255 's in a $3 \times 3$ window. The essential condition for the validity of the window is that at least two pixels in the selected window should be uncorrupted; if not the window size is incremented by 2 . The iteration stops when the window size reaches 7 . In particular case, when the condition for validity doesn't hold in $7 \times 7$ window then the original $3 \times 3$ window is chosen and midpoint of minimum and maximum values of already processed pixels is replaced with the centre pixel. experimental evaluation using MATLAB reveals that our MAUTMPF shows better performance compared to the previous de-noising algorithms in terms of Peak Signal-to-Noise Ratio (PSNR) and Mean Square Error (MSE) for noise densities up to $90 \%$. The validity of the proposed algorithm is verified by testing it for different gray scale images.
\end{abstract}

Keywords: Image Denoising, Salt and Pepper Noise, Median Filter, Unsymmetrical Trimmed Mid-Point Fiter

\section{INTRODUCTION}

Digital images are often corrupted by impulse noise during acquisition, storage and transmission. The impulse noise can be classified under: Salt and pepper noise and random valued noise. The pixel which takes either maximum or minimum intensity value $(0$ or 255$)$ is classified as salt and pepper noise and if the pixel takes any value between 0 and 255 is classified as random valued impulse noise. Though there are various algorithms for removal of salt and pepper noise, they are not efficient at higher noise densities. So we concentrate on the design of efficient algorithm for removal of salt and pepper noise in images particularly for high noise densities.

Several algorithms have been proposed to date for removal of salt and pepper noise in images. Hwang and Hadded (1995) devised an Adaptive Median Filter (AMF).
Astola and Kuosmaneen (1997) proposed standard median filter, Zhang and Karim (2002), Ng and Ma (2006) proposed switching median filters for denoising digital images corrupted by salt and pepper noise.

The above filtering algorithms preserves edge details and show good performance for low noise densities, however they show very poor performance and blur images for noise densities above $50 \%$.

For high noise densities Srinivasan and Ebenezer (2007) proposed Decision Based Algorithm (DBA). The algorithm processes only the noisy pixels and uses a window size of $3 \times 3$. However the filter produces streaking effect for noise densities above $70 \%$. To overcome this (Aiswarya et al., 2010) proposed Decision Based Unsymmetric Trimmed Median Filter (DBUTMF). However the algorithm doesn't perform well for noise densities above $80 \%$.

Corresponding Author: Saravanakumar, S., Department of Electronics and Communication Engineering, Anna University Regional Centre, Coimbatore, India 
$\mathrm{Wu}$ and Tang (2011) use two stage scheme for removal of salt and pepper noise in images. The algorithm uses a detection stage and in painting. In detection stage the difference between pixel in candidate and its neighbours is estimated. If the difference is above threshold the candidate pixel is declared as noisy. The noisy candidate is replaced by Total Variation Inpainting (TVI) method. However the algorithm show poor performance for high noise densities above $90 \%$.

Esakkirajan et al. (2011) proposed a Modified Decision Based Unsymmetric Trimmed Median Filter (MDBUTMF) algorithm especially for images corrupted with high noise densities. In case of highly corrupted images, if all the pixels in processing window are corrupted, the algorithm replaces the centre pixel with the mean of the processing window which blurs the image at high noise densities.

Jourabloo et al. (2012) designed an adaptive median filter for removal of impulse noises in highly corrupted images. The algorithm uses a combination of median filtering and values calculated by its neighbourhood pixels. However the algorithm show poor performance compared to Wu and Tang (2012).

Senthilkumar and Umamaheswari (2012) proposed a new computer aided detection method for the effective detection of breast cancer. Selective median filter is used for noise reduction and Modified Local Range Modification (MLRM) is used for the enhancement. This detection method is found to be performing well and found the detection accuracy of more than 98\%. Nasri et al. (2013) and Zhang et al. (2013) proposed decision based mean filters for removal of salt and pepper noises. The algorithms use non-local windows for estimating the intensity value of pixel in candidate. The filter shows better PSNR values for noise densities above $50 \%$. Vijaykumar et al. (2008) proposed a robust estimation based filter, which reduces the streaking effect but for higher noise densities the image gets blurred. Majhi and Fathi (2005) proposed a novel method based on neurodetector using functional link artificial neural network. The neural detector is based on the concept of training and it detects the impulse noise efficiently, however the training method must be precisely done. An improved spatial filtering technique is adopted for restoration. Mohamed et al. (2010) presented a new Edge Preserving Impulse Noise Filter based on Particle Swarm Optimization. The filter weights were adapted and optimized directionally to restore a corrupted pixel in a mean square sense. The major drawback is the number of population used which restricts the performance for higher noise densities.
In this study we propose a MAUTMPF where we trim the corrupted pixels in a selected window and select the mid-point of the minimum and maximum pixel value of remaining uncorrupted pixels with the essential condition those atleast two pixels in the selected window should be uncorrupted. If not, the size of the window is increased. The iterations in the proposed algorithm is stopped when the size of the window reaches 7 .

The rest of the study is structured as follows. A brief introduction of the proposed filter is given in section 2 . The detailed description of the MAUTMPF with an illustration is presented in section 3. Experimental results and its discussion of the proposed MAUTMPF is presented in section 4. Section 5 gives a brief conclusion about the work done.

\section{PROPOSED FILTER}

The unsymmetrical trimmed filter replaces the noisy pixels in a selected window by taking either mean (Mean Filter) or median (Median Filter) of the remaining pixels after trimming 0 and 255. However, the mean filter blurs the image both at low and high noise densities and median filter doesn't preserve edges for higher noise density. In this brief we propose a MAUTMPF. In the proposed MAUTMPF $3 \times 3$ window is selected and the corrupted pixels are trimmed unsymmetrically. If the remaining pixels in the selected window are greater than or equal to 2 , then the midpoint of maximum and minimum value is taken and replaced by the centre pixel. On the other hand if the remaining pixels in the selected window is less than 2 , then the window size is increased by 2 and same procedure is repeated. The iterations in the algorithm are continued till window size reaches $7 \times 7$. If an estimate for noisy pixel can't be reached in a $7 \times 7$ window also, then the centre pixel is replaced by midpoint of minimum and maximum intensity values of already processed pixels in the initial selected $3 \times 3$ window.

\subsection{MAUTMPF ALGORITHM}

The proposed MAUTMPF processes the corrupted image by detecting all the pixels whether it is corrupted by impulse noise ( 0 or 255$)$. If the pixel is found to be corrupted, then the particular pixel is processed by taking it as the centre pixel of the selected $3 \times 3$ window. The centre pixel is the processing pixel and the noise in the processing pixel is removed by MAUTMPF using the methodology discussed in section 2 .

The flowchart representation of detailed working of the MAUTMPF is given in Fig. 1. 


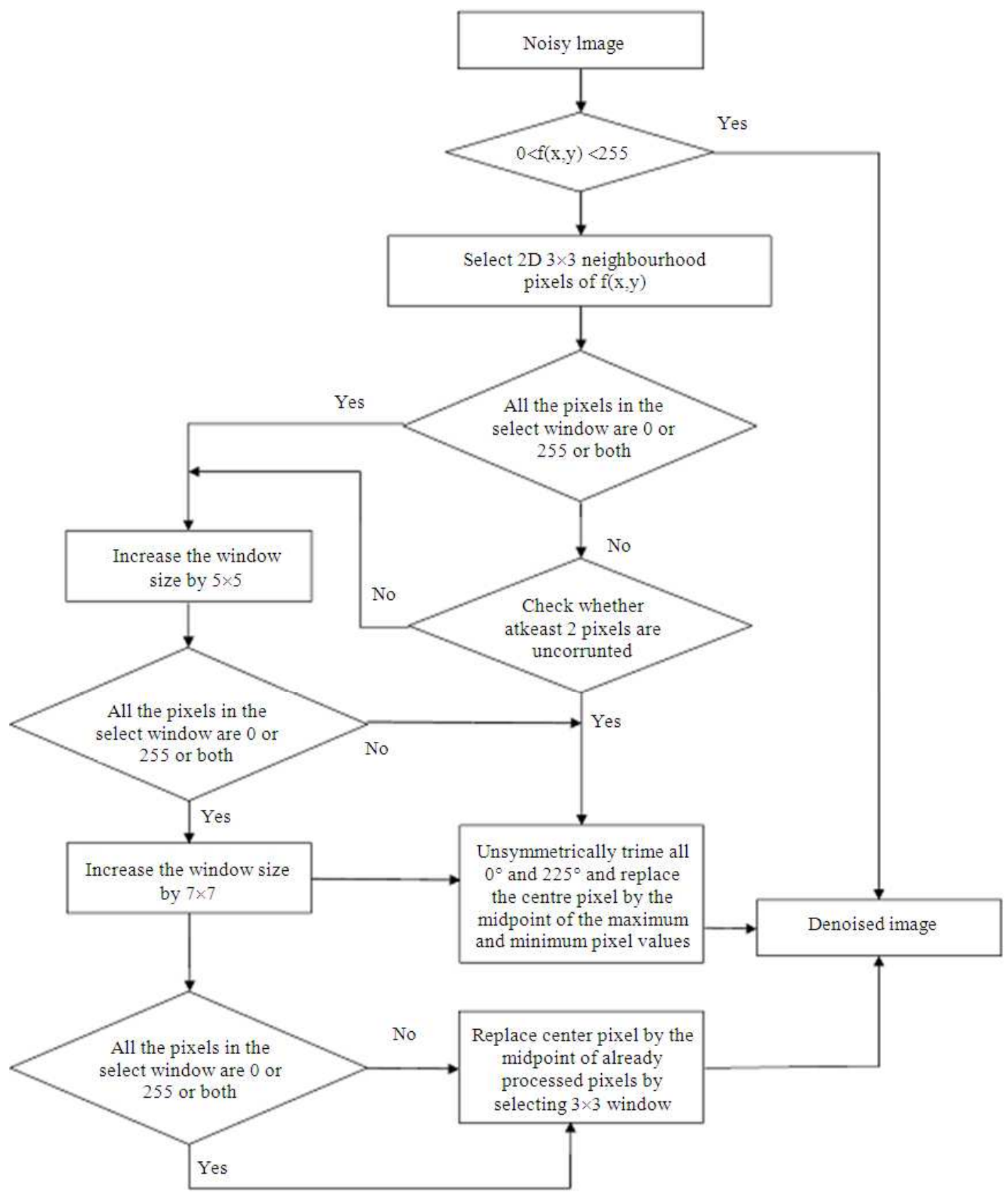

Fig. 1. Flowchart of the proposed MAUTMPF algorithm

\section{ILLUSTRATION OF MAUTMPF ALGORITHM}

The processing of the algorithm starts from the first pixel and moves pixel by pixel till all the pixels in the image are covered. The iterations in the MAUTMPF algorithm are discussed in different cases.

\section{Case 1:}

If the processing pixel (represented as $\mathrm{Pij}$ ) is noisy and the number of uncorrupted pixels in selected $3 \times 3$ window is greater than 2 . 


\section{Case 2:}

If the processing pixel is noisy and the number of uncorrupted pixels in selected $3 \times 3$ window is less than 2 , window size is increased by 2 .

\section{Case 3:}

If the processing pixel is noisy and the number of uncorrupted pixels in the processed $5 \times 5$ window in caseii is less than 2 .

\section{Case 4:}

If the processing pixel is noisy and the number of uncorrupted pixels in the processed $7 \times 7$ window in caseiii is less than 2 .

\section{Case 1:}

About $3 \times 3$ window and the processing pixel is corrupted and there are more than 2 uncorrupted pixels in the selected window:

$\begin{array}{lll}0 & 99 & 110 \\ 0 & 0 & 104 \\ 255 & 255 & 255\end{array}$

Min. value: 99; Max value: 110 Midpoint value $=$ $(99+110) / 2=104.5$ Centre pixel replaced by 104.5:

$\begin{array}{lll}0 & 99 & 110 \\ 0 & 104.5 & 104 \\ 255 & 255 & 255\end{array}$

Actual image value is 104

\section{Case 2:}

Less than 2 uncorrupted pixels in the selected $3 \times 3$ window.

$\begin{array}{lll}255 & 0 & 104 \\ 255 & 0 & 0 \\ 255 & 0 & 255\end{array}$

Only one pixel is uncorrupted, so the window size is incremented by 2 . i.e., consider $5 \times 5$ window.

$\begin{array}{lllll}255 & 255 & 0 & 0 & 255 \\ 255 & 255 & 0 & 104 & 97 \\ 0 & 255 & 0 & 0 & 0 \\ 0 & 255 & 0 & 255 & 110 \\ 97 & 0 & 0 & 0 & 255\end{array}$

Min value: 97; Max value: 110 Midpoint value $=$ $(97+110) / 2=103.5$ Center pixel replaced by 103.5

$\begin{array}{lllll}255 & 255 & 0 & 0 & 255 \\ 255 & 255 & 0 & 104 & 97 \\ 0 & 255 & 103.5 & 0 & 0 \\ 0 & 255 & 0 & 255 & 110 \\ 97 & 0 & 0 & 0 & 255\end{array}$

Actual image value is 104

\section{Case 3:}

Less than 2 uncorrupted pixels in the selected $5 \times 5$ window:

$\begin{array}{lllll}0 & 0 & 0 & 0 & 0 \\ 255 & 255 & 255 & 0 & 255 \\ 0 & 0 & 255 & 0 & 255 \\ 255 & 115 & 255 & 255 & 0 \\ 0 & 255 & 255 & 255 & 255\end{array}$

Only one pixel is uncorrupted, so the window size is incremented by 2 . i.e., consider $7 \times 7$

$\begin{array}{lllllll}255 & 255 & 0 & 0 & 0 & 0 & 0 \\ 255 & 0 & 255 & 0 & 0 & 0 & 255 \\ 0 & 255 & 208 & 0 & 0 & 0 & 255 \\ 0 & 0 & 0 & 255 & 0 & 255 & 219 \\ 0 & 0 & 0 & 255 & 0 & 215 & 0 \\ 255 & 0 & 0 & 0 & 0 & 207 & 0 \\ 255 & 0 & 0 & 0 & 0 & 204 & 0\end{array}$

Min value: 204; Max value: 219 Midpoint value $=$ $(204+219) / 2=211.5$ Center pixel replaced by 211.5

$\begin{array}{lllllll}255 & 255 & 0 & 0 & 0 & 0 & 0 \\ 255 & 0 & 255 & 0 & 0 & 0 & 255 \\ 0 & 255 & 208 & 0 & 0 & 0 & 255 \\ 0 & 0 & 0 & 211.5 & 0 & 255 & 219 \\ 0 & 0 & 0 & 255 & 0 & 215 & 0 \\ 255 & 0 & 0 & 0 & 0 & 207 & 0 \\ 255 & 0 & 0 & 0 & 0 & 204 & 0\end{array}$

Actual image value is 210

\section{Case 4:}

Less than 2 uncorrupted pixels in the selected $7 \times 7$ window: 


$\begin{array}{lllllll}0 & 0 & 0 & 0 & 0 & 0 & 0 \\ 0 & 0 & 255 & 255 & 255 & 0 & 255 \\ 0 & 0 & 0 & 255 & 0 & 255 & 0 \\ 255 & 0 & 0 & 255 & 0 & 255 & 255 \\ 0 & 0 & 255 & 0 & 255 & 0 & 255 \\ 0 & 255 & 255 & 0 & 0 & 0 & 0 \\ 255 & 255 & 0 & 0 & 255 & 255 & 255 \\ & & & & & & \\ 104 & & & 103 & & & 102 \\ 101 & & & 255 & & & 0 \\ 255 & & & 0 & & & 255\end{array}$

Min value: 101; Max value: 104 Midpoint value = $(101+104) / 2=102.5$ Center pixel replaced by 102.5

$\begin{array}{lll}104 & 103 & 102 \\ 101 & 102.5 & 0 \\ 255 & 0 & 255\end{array}$

Actual image value is 103 .

\section{RESULTS AND DISCUSSION}

The performance of the proposed MAUTMPF is tested with different gray scale images. The noise density (intensity) is varied from $10 \%$ to $90 \%$. De-noising performances are quantitatively measured by the PSNR and Mean Square Error (MSE) as defined in Equation 1 and 2 respectively:

PSNR in $\mathrm{dB}=10 \log _{10}\left(\frac{255^{2}}{\mathrm{MSE}}\right)$

$\operatorname{MSE}=\frac{\sum_{i} \sum_{j}(Y(i, j)-\bar{Y}(i, j))^{2}}{M X N}$

\author{
Where: \\ M X N = Size of the image \\ $\mathrm{Y} \quad=$ Represents the original image \\ $\overline{\mathrm{Y}} \quad=$ Denotes the de-noised image
}

The PSNR and MSE values of the proposed MAUTMPF and previous researches for varying the noise density from 10 to $90 \%$ are shown in Table $\mathbf{1}$ and 2 respectively. From the PSNR values it is seen that our proposed MAUTMPF demonstrates PSNR improvement of $73.79,45.50,48.86,26.00,24.93$ and $17.65 \%$ for noise density of $30 \%$ and $227.79,113.44,174.92,51.61$, 44.86 and $33.95 \%$ compared to MF, AMF, PSMF, DBA, MDBA and MDBUTMF algorithms respectively. The proposed MAUTMPF demonstrates MSE reduction of 95.6, 87.6, 76.2, 81.6, 64.5 and $60.8 \%$ for $30 \%$ noise density and 98.4,92.3, 95.4,85.7, 63.8 and $49.4 \%$ for $70 \%$ noise density compared to MF, AMF, PSMF, DBA, MDBA and MDBUTMF algorithms respectively when tested against Lena image. From the results it can be seen that the proposed MAUTMPF demonstrates better PSNR improvement and MSE reduction compared to the existing algorithms both at high and low noise densities.

In addition we have tested the performance of the proposed algorithm against Pepper, Baboon, Camera man and Boat images at 30 and $70 \%$ noise densities and shown in Table 3. The results are compared with MF, AMF, PSMF, DBA, MDBA and MDBUTMF algorithms. It is seen that the proposed algorithm outperforms all other previous algorithms in terms of PSNR and MSE irrespective of the nature of image. The output pepper image processed by our MAUTMPF algorithm for noise densities 10 to $90 \%$ is shown in Fig. 2.

Table 1. Comparison of PSNR values of different algorithms for Lena image at different noise densities

\begin{tabular}{|c|c|c|c|c|c|c|c|}
\hline \multirow[b]{2}{*}{ Noise density $(\%)$} & \multicolumn{7}{|c|}{ PSNR in $\mathrm{dB}$} \\
\hline & MF & AMF & PSMF & DBA & MDBA & MDBUTMF & Proposed MAUTMPF \\
\hline 10 & 26.34 & 28.43 & 30.22 & 36.40 & 36.94 & 37.91 & 42.53 \\
\hline 20 & 25.66 & 27.40 & 28.39 & 32.90 & 32.69 & 34.78 & 39.50 \\
\hline 30 & 21.86 & 26.11 & 25.52 & 30.15 & 30.41 & 32.29 & 37.99 \\
\hline 40 & 18.21 & 24.40 & 22.49 & 28.49 & 28.49 & 30.32 & 36.56 \\
\hline 50 & 15.04 & 23.36 & 19.13 & 26.52 & 26.52 & 28.18 & 35.37 \\
\hline 60 & 11.08 & 20.60 & 12.10 & 24.41 & 24.41 & 26.43 & 34.13 \\
\hline 70 & 9.93 & 15.25 & 11.84 & 21.47 & 22.47 & 24.30 & 32.55 \\
\hline 80 & 8.68 & 10.31 & 8.020 & 20.44 & 20.44 & 21.70 & 30.70 \\
\hline 90 & 6.65 & 7.930 & 6.570 & 17.56 & 17.56 & 18.40 & 28.17 \\
\hline
\end{tabular}


Saravanakumar, S. et al. / Journal of Computer Science 10 (7): 1307-1314, 2014

Table 2. Comparison of MSE values of different algorithms for lena image at different noise densities

\begin{tabular}{|c|c|c|c|c|c|c|c|}
\hline \multirow[b]{2}{*}{ Noise density (\%) } & \multicolumn{7}{|l|}{ MSE } \\
\hline & MF & AMF & PSMF & DBA & MDBA & MDBUTMF & Proposed MAUTMPF \\
\hline 10 & 27.560 & 33.76 & 24.9000 & 20.64 & 9.300 & 4.56 & 3.63 \\
\hline 20 & 81.126 & 36.60 & 37.6033 & 38.56 & 18.600 & 17.12 & 7.29 \\
\hline 30 & 256.320 & 83.53 & 43.4000 & 56.10 & 29.100 & 26.34 & 10.33 \\
\hline 40 & 305.200 & 125.66 & 203.4500 & 81.36 & 35.400 & 30.40 & 14.35 \\
\hline 50 & 677.050 & 147.34 & 352.1400 & 113.12 & 41.580 & 41.23 & 18.87 \\
\hline 60 & 1330.060 & 254.77 & 478.8500 & 163.84 & 130.700 & 61.25 & 25.13 \\
\hline 70 & 2241.230 & 466.56 & 789.2300 & 251.85 & 99.960 & 71.50 & 36.15 \\
\hline 80 & 3464.500 & 517.56 & 2205.3200 & 305.39 & 169.610 & 118.90 & 55.36 \\
\hline 90 & 4883.210 & 1041.99 & 3987.3500 & 730.72 & 240.925 & 134.00 & 99.00 \\
\hline
\end{tabular}

Table 3. Comparison of PSNR values of different algorithms for lena, pepper, baboon, cameraman and boat images at noise densities $30 \%$ and $70 \%$

\begin{tabular}{lllllllll}
\hline \multirow{2}{*}{ Noise level } & Image & MF & AMF & PSMF & DBA & MDBA & MDBUTMF & MAUTMPF \\
\hline $30 \%$ & Lena & 21.86 & 26.11 & 25.52 & 30.15 & 30.41 & 32.29 & 37.99 \\
& Pepper & 19.26 & 31.82 & 31.25 & 32.86 & 31.25 & 31.59 & 34.92 \\
& Baboon & 18.65 & 21.66 & 24.12 & 24.38 & 22.39 & 24.61 & 26.79 \\
& Camera man & 17.69 & 19.37 & 20.58 & 22.62 & 24.59 & 25.91 & 30.12 \\
$70 \%$ & Boat & 17.57 & 28.05 & 29.64 & 27.84 & 28.99 & 30.05 & 33.37 \\
& Lena & 9.93 & 15.25 & 11.84 & 21.47 & 22.47 & 24.30 & 32.55 \\
& Pepper & 9.52 & 18.72 & 14.56 & 17.02 & 21.87 & 25.24 & 29.48 \\
& Baboon & 10.11 & 14.86 & 10.05 & 20.55 & 20.54 & 20.80 & 21.88 \\
& Camera man & 9.46 & 13.93 & 9.47 & 20.84 & 19.97 & 22.52 & 25.53 \\
& Boat & 12.8 & 15.24 & 15.23 & 16.56 & 18.88 & 21.37 & 28.09 \\
\hline
\end{tabular}

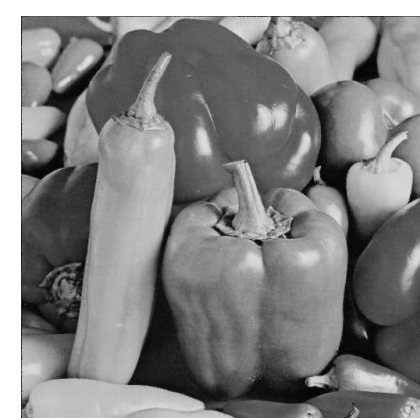

(a)

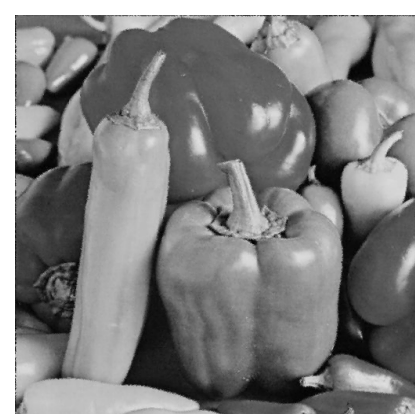

(e)

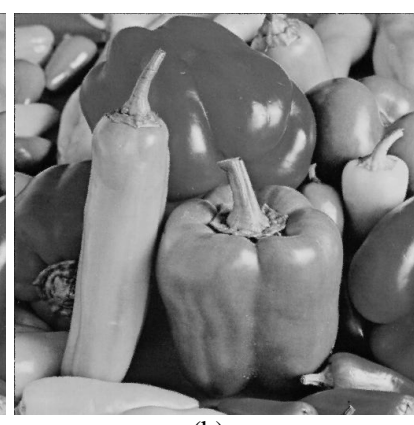

(b)

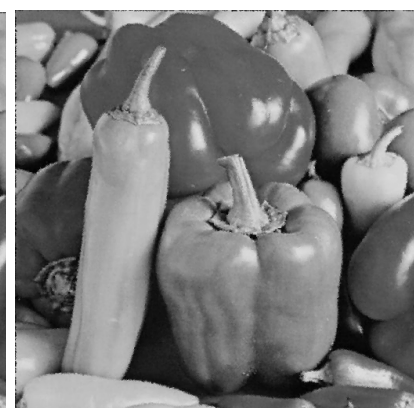

(f)

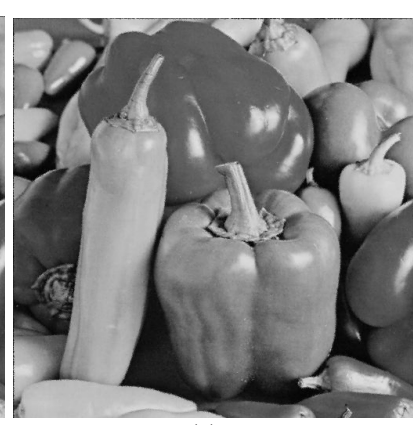

(c)

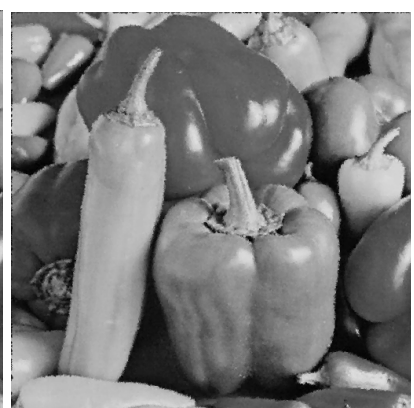

(g)

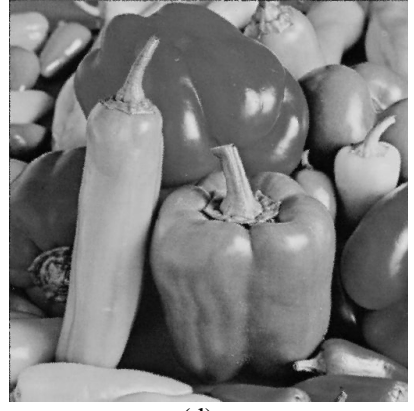

(d)

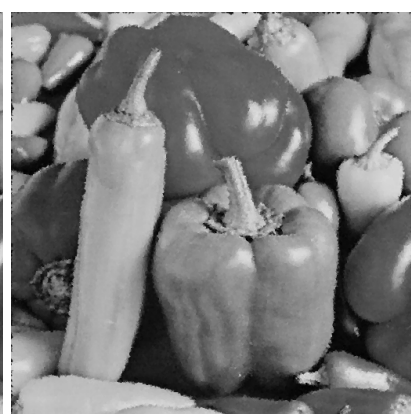

(h) 


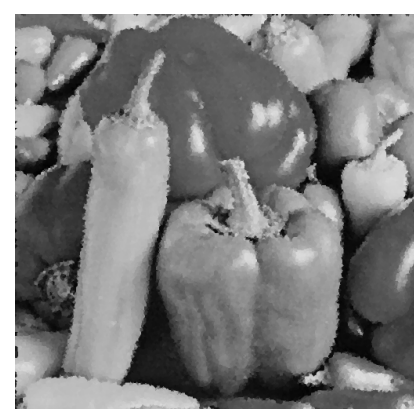

(i)

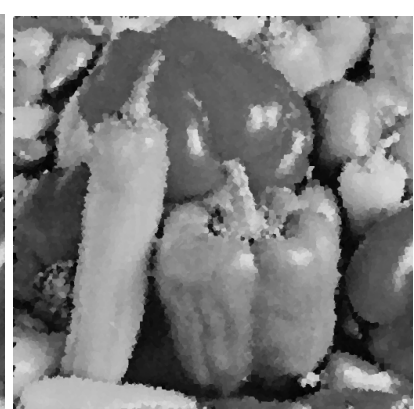

(j)

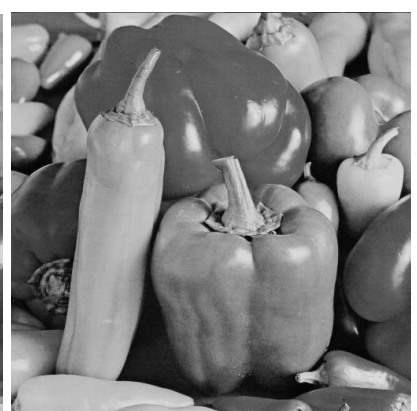

(k)

Fig. 2. Output Pepper images (a)-(j) processed by MAUTMPF algorithm for noise densities 10 to $95 \%$ respectively, (k) original image
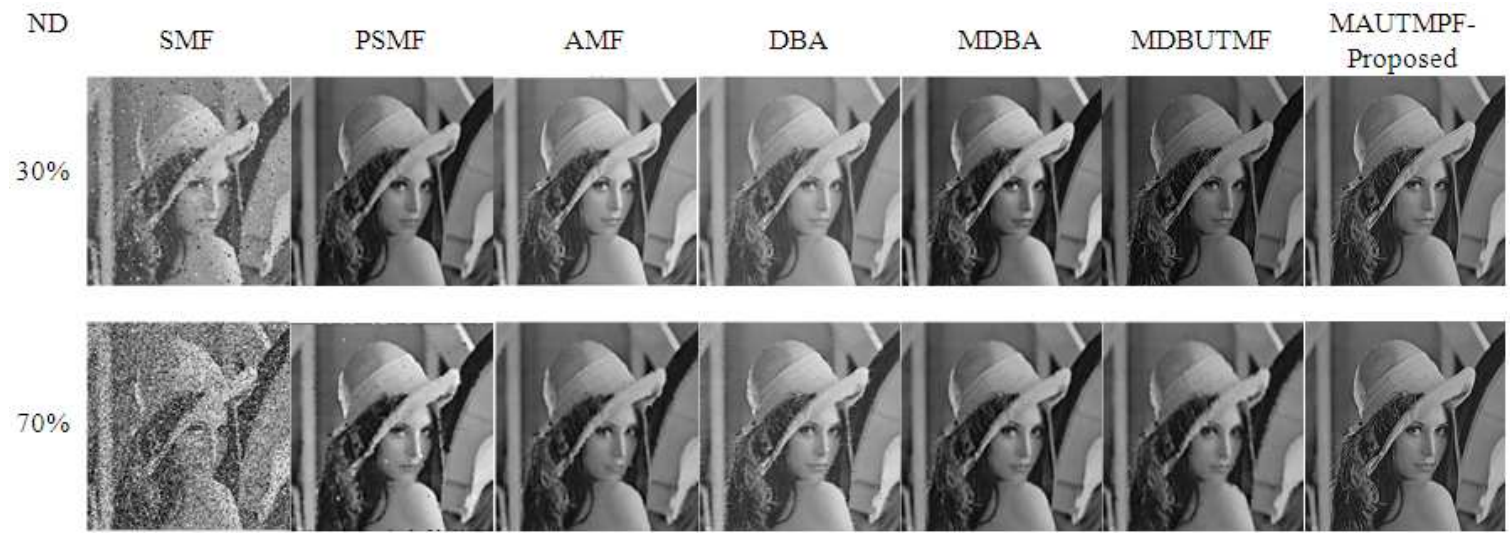

Fig. 3. Output images of proposed MAUTMPF and existing algorithms of lena for 30 and $70 \%$ noise densities

The qualitative analysis of our MAUTMPF is performed by comparing the output Lena images processed by MAUTMPF algorithm and existing algorithms for two different noise densities (low-30\% and high-70\%) and are shown in Fig. 3. In Fig. 3, the first row represents the type of algorithm, second row represents output images processed by different algorithms for $30 \%$ noise density and third row represents the output images processed by different algorithms for $70 \%$ noise density. From the Fig. 3, it is possible to observe that the quality of the restored image using MAUTMPF is better than the quality of the restored image using existing algorithms.

\section{CONCLUSION}

In this study, we have proposed a MAUTMPF algorithm for de-noising images corrupted by impulse noise. Experimental evaluation of the proposed MAUTMPF algorithm demonstrates better performance in terms of PSNR and MSE values compared to previous algorithms. The performance of our algorithm is tested against varying noise densities and also against different images. The numerical and visual results entail that our algorithm outperforms against different noise densities irrespective of the nature of the image compared to prior arts.

\section{REFERENCES}

Aiswarya, K., V. Jayaraj and D. Ebenezer, 2010. A new and efficient algorithm for the removal of high density salt and pepper noise in images and videos. Proceedings of the 2nd International Conference on Computer Modeling and Simulation, Jan. 22-24, IEEE Xplore Press, Sanya, Hainan, pp: 409-413. DOI: $10.1109 /$ ICCMS.2010.310

Astola, J. and P. Kuosmaneen, 1997. Fundamentals of Nonlinear Digital Filtering. 1st Edn., Taylor and Francis, ISBN-10: 0849325706, pp: 288. 
Esakkirajan, S., T. Veerakumar, A.N. Subramanyam and C.H.P. Chand, 2011. Removal of high density salt and pepper noise through modified decision based unsymmetric trimmed median filter. IEEE Signal Proc. Lett., 5: 287-290. DOI: 10.1109/LSP.2011.2122333

Hwang, H. and R.A. Hadded, 1995. Adaptive median filter: New algorithms and results. IEEE Trans. Image Proc., 4: 499-502. DOI: 10.1109/83.370679

Wu, J. and C. Tang, 2011. An efficient decision-based and edge-preserving method for salt-and-pepper noise removal. Patt. Recogn. Lett., 15: 1974-1981. DOI: 10.1016/j.patrec.2011.09.025

$\mathrm{Wu}$, J. and C. Tang, 2012. A new filter for the removal of random-valued impulse noise from highly corrupted images. AUE-Int. J. Electron. Commun., 10: 847-854. DOI: 10.1016/j.aeue.2012.03.002

Jourabloo, A.H. Feghahati and M. Jamzad, 2012. New algorithms for recovering highly corrupted images with impulse noise. Scientia Iranica, 19: 1738-2012. DOI: 10.1016/j.scient.2012.07.016

Majhi, J. and M. Fathi, 2005. FLANN detector based filtering of images corrupted by impulse noise. J. Comput. Sci., 3: 332-336. DOI: 10.3844/jcssp.2005.332.336

Mohamed, S., M. Roomi, P. Rajesh and B.G. Revathi, 2010. A particle swarm optimization based edge preserving impulse noise filter. J. Comput. Sci., 9: 1014-1020.
Nasri, M., S. Saryazdi and H. Nezamabadi-Pour, 2013. SNLM: A switching non-local means filter for removal of high density salt and pepper noise. Scientia Iranica, 3: 760-764. DOI: 10.1016/j.scient.2013.01.001

Ng, P.E. and K.K. Ma, 2006. A switching median filter with boundary discriminative noise detection for extremely corrupted images. IEEE Trans. Image Proc., 6: 1506-1516. DOI: 10.1109/TIP.2005.871129

Senthilkumar, B. and G. Umamaheswari, 2012. New computer aided detection method for the effective detection of breast cancer. OnLine J. Biol. Sci., 12: 156-160. DOI: 10.3844/ojbsci.2012.156.160

Srinivasan, K.S. and D. Ebenezer, 2007. A new fast and efficient decision-based algorithm for removal of high-density impulse noises. IEEE Sig. Proc. Lett., 3: 189-192. DOI: 10.1109/LSP.2006.884018

Vijaykumar, V.R., P.T.Vanathi, P. Kanagasabapathy and D. Ebenezer, 2008. High density impulse noise removal using robust estimation based filter. IAENG Int. J. Comput. Sci., 3: 1-10.

Zhang, X., Y. Zhan, M. Ding, W. Hou and Z. Yin, 2013. Decision-based non-local means filter for removing impulse noise from digital images, Signal Proc., 2: 517-524. DOI: 10.1016/j.sigpro.2012.08.022

Zhang, S. and M.A. Karim, 2002. A new impulse detector for switching median filters. IEEE Signal Proc. Lett., 11: 360-363. DOI: 10.1109/LSP.2002.805310 